\title{
On the Generators of Quantum Dynamical Semigroups
}

\author{
G. Lindblad \\ Department of Theoretical Physics, Royal Institute of Technology, \\ S-100 44 Stockholm 70, Sweden
}

\begin{abstract}
The notion of a quantum dynamical semigroup is defined using the concept of a completely positive map. An explicit form of a bounded generator of such a semigroup on $B(\mathscr{H})$ is derived. This is a quantum analogue of the Lévy-Khinchin formula. As a result the general form of a large class of Markovian quantum-mechanical master equations is obtained.
\end{abstract}

\section{Introduction}

The dynamics of a finite closed quantum system is conventionally represented by a one-parameter group of unitary transformations in Hilbert space. This formalism makes it difficult to describe irreversible processes like the decay of unstable particles, approach to thermodynamic equilibrium and measurement processes [1-3].

It seems that the only possibility of introducing an irreversible behaviour in a finite system is to avoid the unitary time development altogether by considering non-Hamiltonian systems. One way of doing this is by postulating an interaction of the considered system $S$ with an external system $R$ like a heat bath or a measuring instrument. This approach is suggested by the theory of the measurement process in quantum theory, which provides an example of an irreversible process even in the axioms of quantum theory, and by Einstein's theory of Brownian motion where the fluid provides a stochastic external force which determines the irreversible nature of the motion. A different physical interpretation with the same mathematical structure is to consider $S$ as a limited set of (macroscopic) degrees of freedom of a large system $S+R$ and $R$ as the uncontrolled (microscopic) degrees of freedom. If the reservoir $R$ is supposed to be finite (but large) then the development of the system $S+R$ may be given by a unitary group of transformations. The partial state of $S$ then suffers a time development which is not given by a unitary transformation in general.

The simplest dynamics for $S$ which could describe a genuinely irreversible process is a semigroup of transformations which introduces a preferred direction 
in time. It is difficult, however, to give physically plausible conditions on the system $S+R$ which rigorously imply a semigroup law of motion for the subsystem $S$. Intuitively the time scale considered for $S$ should be very long compared with the relaxation time of the external system $R$, but shorter than the recurrence time for the system $S+R$ considered as a closed finite system. There is thus only an intermediate time interval in which the semigroup behaviour is approximated. In order to obtain an exact semigroup law for $S$ it is necessary (but not sufficient) to let the size of $R$ approach infinity.

The only rigorous treatments of this problem known to the author were given by Davies for a model of a harmonic oscillator [4] and for a $\mathrm{N}$-level atom [5] and by Pulè for a spin $\frac{1}{2}$ system [6]. In each case the system is in contact with an infinite heat bath. The results are that the weak coupling limits of the time developments are Markovian for large classes of interaction Hamiltonians. The argument for choosing the set of dynamical maps to form a semigroup must, however, be based mostly on the simplicity and the success of physical applications. Applications to processes like laser action, spin relaxation etc., of Markovian master equations, where the Liouville operator is just the generator of the dynamical semigroup, have led some authors to introduce the semigroup law as the fundamental dynamical postulate for open (non-Hamiltonian) systems [7-9].

The axioms for a dynamical semigroup as given by Ingarden and Kossakowski $[8,9]$ will be repeated here in the Heisenberg picture.

Let $\mathscr{A}$ be a $W^{*}$-algebra. A dynamical semigroup is a one-parameter family $\Phi_{t}$ of maps of $\mathscr{A}$ into itself satisfying

a) $\Phi_{t}$ is positive

b) $\Phi_{t}(I)=I$

c) $\Phi_{s} \cdot \Phi_{t}=\Phi_{s+t}$

d) $\Phi_{t}(X) \rightarrow X$ ultraweakly, $t \rightarrow 0$

e) $\Phi_{t}$ is normal (ultraweakly continuous).

Then we know that there is a (generally unbounded) map $L$ defined on an ultraweakly dense domain such that

$$
\lim _{t \downarrow 0}\left\|L X-t^{-1}\left(\Phi_{t} X-X\right)\right\|=0
$$

for $X$ in the domain [10].

The purpose of this paper is to derive an explicit form for the generators of a dynamical semigroup. In order to obtain the desired result we have to introduce two restrictions in the class of dynamical semigroups compared with the scheme outlined above.

First we have to assume that the semigroup is norm continuous, that is that the generator is a bounded map ([11], p. 341), a condition which is not fulfilled in many applications. (We may hope that this restriction can be ultimately removed using more powerful mathematics.) This means that $\mathrm{d}$ ) is replaced by

$\left.\mathrm{d}^{\prime}\right) \lim _{t \downarrow}\left\|\Phi_{t}-I\right\|=0$.

It then follows that there is a bounded map $L: \mathscr{A} \rightarrow \mathscr{A}$ such that

f) $\Phi_{t}=\exp t L$

g) $\lim _{t \downarrow 0}\left\|L-t^{-1}\left(\Phi_{t}-I\right)\right\|=0$. 
From the fact that the set of ultraweakly continuous linear functionals on $\mathscr{A}$ is norm closed it follows that the set of ultraweakly continuous maps of $\mathscr{A}$ into itself is norm closed ([12], 1.3.3). Consequently

h) $L$ is ultraweakly continuous.

The second restriction is that we have to assume the dynamical maps to be not merely positive but completely positive (CP) in the sense of Stinespring [13], i.e., using the notation of $\S 2$, we replace a) by

a') $\Phi_{t} \in \mathrm{CP}(\mathscr{A})$.

This we believe to be a physically motivated hypothesis. $\S 2$ is devoted to a justification of this statement and a description of those mathematical properties of $\mathrm{CP}$ maps which are essential for the following paragraphs.

In $\S 3$ the generators of $\mathrm{CP}$ semigroups, here called completely dissipative (CD) maps, are introduced as well as a dissipation function which describes the deviation from Hamiltonian dynamics. The canonical form for the CD generators is derived in $\S 4$ in terms of a CP map and a Hamiltonian. The set of CD generators form a convex cone. In $\S 5$ this convex structure is described and the extreme rays of the cone are given. The results of $\S 4$ and $\S 5$ provide a quantum analogue of the classical Lévy-Khinchin formula [14]. In $\S 6$ the connections with previous work is described further.

\section{Completely Positive Maps}

At first sight it is a surprising fact that the property of positivity is not sufficient for a dynamical map to have a physical interpretation for quantum systems, and that we must demand the stronger property of complete positivity. In order to see the physical background of this we consider two quantum systems $S_{1}$ and $S_{2}$ and a heat bath $R$. We assume that $S_{1}$ is interacting with $R$ in such a way that the time development of $S_{1}$ is described by a family of maps

$$
\Phi_{1, t}: B\left(\mathscr{H}_{1}\right) \rightarrow B\left(\mathscr{H}_{1}\right)
$$

where $\mathscr{H}_{i}$ is the Hilbert space belonging to $S_{i}$. We can consider one fixed time and drop the time parameter. We further assume that $S_{2}$ is a closed system, i.e. its dynamics is given by a Hamiltonian $\mathrm{H}_{2}$. We put $\mathrm{H}_{2}=0$ for the moment. Then we ask: can the map $\Phi_{1}$ be extended to a positive map $\Phi: B(\mathscr{H}) \rightarrow B(\mathscr{H})$ where $\mathscr{H}=\mathscr{H}_{1} \otimes \mathscr{H}_{2}$, such that $S_{2}$ is unaffected? This is obviously so when the dynamics of $S_{1}$ is Hamiltonian. Then the dynamics of $S_{1}+S_{2}$ is given by the Hamiltonian $H=H_{1} \otimes I_{2} . \Phi$ is defined by

$$
\Phi(X \otimes Y)=\Phi_{1}(X) \otimes Y
$$

all $X \in B\left(\mathscr{H}_{1}\right), \quad Y \in B\left(\mathscr{H}_{2}\right)$, or more explicitly by $\Phi(X)=U^{+} X U$ where $U=$ $\exp \left(i H_{1} t\right) \otimes I_{2}$, and $\Phi$ is obviously positive. In the case where the dynamics of $S_{1}$ is non-Hamiltonian (2.1) follows from the conditions

$$
\Phi(X \otimes I)=\Phi_{1}(X) \otimes I, \quad \Phi(I \otimes Y)=I \otimes Y,
$$

but the positively of $\Phi$ is not obvious from a mathematical point of view though necessary for physical reasons. Let first $\mathscr{H}_{2}$ have finite dimension $n$. Then $B(\mathscr{H}) \cong$ 
$M_{n}\left(B\left(\mathscr{H}_{1}\right)\right)$ where $M_{n}(\mathscr{A})$ denotes the $n \times n$ matrix algebra over a $C^{*}$-algebra $\mathscr{A}$. Consider a more general case by introducing two $C^{*}$-algebras $\mathscr{A}, \mathscr{B}$. If $\Phi$ is a linear map from $\mathscr{A}$ into $\mathscr{B}$ then $\Phi$ extends to a map $\Phi_{n}: M_{n}(\mathscr{A}) \rightarrow M_{n}(\mathscr{B})$ defined by

$$
\Phi_{n}\left(X \otimes E_{i j}\right)=\Phi(X) \otimes E_{i j}
$$

where $E_{i j}, i, j=1, \ldots, n$ are matrix units spanning $M_{n}(\mathbb{C})$. We write this as $\Phi_{n}=\Phi \otimes I_{n}$. When $\mathscr{A}=\mathscr{B}=\mathscr{B}\left(\mathscr{H}_{1}\right)$ then $(2.2)$ is (2.1).

Now $\Phi_{n}$ is not necessarily positive. We must therefore restrict ourselves to maps $\Phi$ such that $\Phi_{n}$ is always positive.

Definition. $\Phi: \mathscr{A} \rightarrow \mathscr{B}$ is called completely positive iff $\Phi_{n}$ is positive for all $n$. We write this as $\Phi \in \mathrm{CP}(\mathscr{A}, \mathscr{B})$ or $\Phi \in \mathrm{CP}(\mathscr{A})$ if $\mathscr{A}=\mathscr{B}$. If $\mathscr{A}=\mathscr{B}=\mathscr{B}(\mathscr{H})$ then we write $\Phi \in \mathrm{CP}(\mathscr{H})$ for simplicity $[13,15,16]$.

Remarks 1. There are positive maps which are not CP $[15,17]$.

2. $\mathrm{CP}(\mathscr{A}, \mathscr{B})$ is a convex cone.

3. $\Phi \in \mathrm{CP}(\mathscr{A}, \mathscr{B}), \Psi \in \mathrm{CP}(\mathscr{B}, \mathscr{C}) \Rightarrow \Psi \cdot \Phi \in \mathrm{CP}(\mathscr{A}, \mathscr{C})$.

Stinespring has given the following canonical form of CP maps [13]. We assume that $\mathscr{A}, \mathscr{B}$ have units troughout.

Lemma 1. $\Phi: \mathscr{A} \rightarrow \mathscr{B} \subset \mathscr{B}(\mathscr{H})$ is $\mathrm{CP}$ iff there is a*-representation $\pi$ of $\mathscr{A}$ in a Hilbert space $\mathscr{K}$ and a bounded linear map $V: \mathscr{H} \rightarrow \mathscr{K}$ such that

$$
\Phi(X)=V^{+} \pi(X) V .
$$

Corollary. If $\Phi \in \mathrm{CP}(\mathscr{A}, \mathscr{B})$ then $\Phi_{n} \in \mathrm{CP}\left(M_{n}(\mathscr{A}), M_{n}(\mathscr{B})\right)$ all $n$.

The proof is obvious.

If $\mathscr{A}, \mathscr{B}$ are $W^{*}$-algebras we will denote by $\mathrm{CP}(\mathscr{A}, \mathscr{B})_{\sigma}$ the set of ultraweakly continuous (normal) elements in $\mathrm{CP}(\mathscr{A}, \mathscr{B})$.

Lemmà 2. $\Phi \in \mathrm{CP}(\mathscr{H})_{\sigma}$ iff

$$
\Phi(X)=\sum V_{i}^{+} X V_{i}
$$

where $V_{i}, \sum V_{i}^{+} V_{i} \in B(\mathscr{H})$.

The proof of this lemma was given by Kraus [18].

It is now easy to see how to generalize the tensor product extension property discussed in the beginning of this paragraph.

Proposition 1. Let $\mathscr{H}=\mathscr{H}_{1} \otimes \mathscr{H}_{2}$ and assume that $\Phi_{\alpha} \in \mathrm{CP}\left(\mathscr{H}_{\alpha}\right)_{\sigma}$ for $\alpha=1,2$. Then there is a unique $\Phi \in \mathrm{CP}(\mathscr{H})_{\sigma}$ such that $\Phi(X \otimes Y)=\Phi_{1}(X) \otimes \Phi_{2}(Y)$, all $X \in B\left(\mathscr{H}_{1}\right)$, $Y \in B\left(\mathscr{H}_{2}\right)$.

Proof. From Lemma 2 we know that $\Phi_{\alpha}(X)=\sum_{i} V_{\alpha i}^{+} X V_{\alpha i}, \alpha=1,2$. Then obviously

$$
\Phi(X)=\sum_{i, j}\left(V_{1 i}^{+} \otimes V_{2 j}^{+}\right) X\left(V_{1 i} \otimes V_{2 j}\right)
$$

has the stated properties. We write this as $\Phi=\Phi_{1} \otimes \Phi_{2}$.

In addition to this extension property the $\mathrm{CP}$ maps have the important property of being realizable through the interaction of the considered system $S$ with an external system $R$. Let $\varrho_{0}$ be the initial state of the system $R$. If $S+R$ is 
treated as a closed finite system, then its development during a specified time interval will be given by a unitary transformation $U$ in $\mathscr{H}=\mathscr{H}_{S} \otimes \mathscr{H}_{R}$. In the Heisenberg picture the development of $S$ is given by

$$
X \rightarrow \Phi(X)=\operatorname{Tr}_{R}\left[\varrho_{0} U^{+}(X \otimes I) U\right] .
$$

It is evident that $\Phi \in \mathrm{CP}\left(\mathscr{H}_{S}\right)_{\sigma}$ and $\Phi(I)=I$.

We have a converse result. Let $S(\mathscr{H})$ denote the set of normal states on $B(\mathscr{H})$.

Proposition 2. If $\Phi \in \mathrm{CP}\left(\mathscr{H}_{1}\right)_{\sigma}, \Phi(I)=I$ then there is an isometric operator $V$ in $\mathscr{H}=\mathscr{H}_{1} \otimes \mathscr{H}_{2}$, where $\mathscr{H}_{2}$ is some Hilbert space, such that for all $\varrho \in S\left(\mathscr{H}_{2}\right)$

$$
\Phi(X)=\operatorname{Tr}_{2}\left(\varrho V^{+}(X \otimes I) V\right) .
$$

Proof. We know from Lemma 2 that

$$
\Phi(X)=\sum V_{i}^{+} X V_{i}, \quad \sum V_{i}^{+} V_{i}=I .
$$

When $\mathscr{H}_{2}$ is $\infty$-dimensional there are isometries $W_{i}$ in $\mathscr{H}_{2}$ such that $W_{i}^{+} W_{j}=$ $\delta_{i j} I$. Define $V=\sum V_{i} \otimes W_{i}$. Then

$$
V^{+}(X \otimes I) V=\sum V_{i}^{+} X V_{i} \otimes W_{i}^{+} W_{i}=\Phi(X) \otimes I .
$$

Remark. $V$ can be extended to a unitary operator $U$ in a larger Hilbert space $\mathscr{H}^{\prime}$ [19]. Then the statement holds with $U$ replacing $V$ for some $\varrho$ in $S\left(\mathscr{H}^{\prime}\right)$.

The properties of $\mathrm{CP}$ maps given above seem to be argument enough to postulate the dynamical maps to be CP. Note that for classical systems where the algebra of observables is abelian, positive maps are always CP [13], hence there is no additional restriction in that case.

We introduce a partial order on $\mathrm{CP}(\mathscr{A})$ by:

$\Psi \leqq \Phi \quad$ iff $\quad \Phi-\Psi \in \mathrm{CP}(\mathscr{A})$.

Definition. $\Phi \in \mathrm{CP}(\mathscr{A})$ is pure (belongs to an extreme ray) iff $\Psi \in \mathrm{CP}(\mathscr{A}), \Psi \leqq \Phi \Rightarrow$ $\Psi=\lambda \Phi, \lambda \in[0,1]$. We write this $\Phi \in \operatorname{extr} \mathrm{CP}(\mathscr{A})$.

Lemma 4. $\Phi \in \operatorname{extr} \mathrm{CP}(\mathscr{H})_{\sigma}$ iff $\Phi(X)=V^{+} X V, V \in B(\mathscr{H})$.

Proof. Corollary 1.4.3 of [15] says that $\Phi \in \operatorname{extr} \mathrm{CP}(\mathscr{H})$ iff $\pi$ in (2.3) is an irreducible representation of $B(\mathscr{H})$. Hence if $\pi(X)=X$ then

$$
\Phi \in \mathrm{CP}(\mathscr{H})_{\sigma} \cap \operatorname{extr} \mathrm{CP}(\mathscr{H}) C \operatorname{extr} \mathrm{CP}(\mathscr{H})_{\sigma} .
$$

But from Lemma 2 follows conversely that every $\Phi \in \operatorname{extr} \mathrm{CP}(\mathscr{H})_{\sigma}$ is of the form $V^{+} X V$.

Lemma 5. If $\Phi \in \mathrm{CP}(\mathscr{A})$, where $\mathscr{A}$ is a $C^{*}$-algebra, then for all $X \in \mathscr{A}$

$$
\Phi\left(X^{+}\right) \Phi(I)^{-1} \Phi(X) \leqq \Phi\left(X^{+} X\right) .
$$

When $\Phi(I)=I$ we get

$$
\Phi\left(X^{+}\right) \Phi(X) \leqq \Phi\left(X^{+} X\right)
$$

Proof. (2.6) actually implies (2.5) by the transformation $\Phi(X) \rightarrow \Phi(I)^{-\frac{1}{2}} \Phi(X) \Phi(I)^{-\frac{1}{2}}$. (2.6) is proved in [16] Theorem 3.1. 
Remarks 1. From the corollary of Lemma 1 follows that $\Phi_{n}$ satisfies (2.5) for all $n$.

2. From (2.5) follows that $\|\Phi(U)\| \leqq\|\Phi(I)\|$ if $U$ is unitary. From Corollary 1 of [20] we know that $\|\Phi\|=\sup _{U}\|\Phi(U)\|$ where supremum is over the unitaries in $\mathscr{A}$. Consequently $\|\Phi\|=\|\Phi(I)\|$.

\section{Generators of Completely Positive Semigroups}

In this paragraph we let $\mathscr{A}$ be a $W^{*}$-algebra and $\Phi_{t}=\operatorname{expt} L$ a norm continuous semigroup in $\mathrm{CP}(\mathscr{A})$. At first we will assume that $\Phi_{t}(I)=I$. The extension $L_{n}$ of the generator $L$ to $M_{n}(\mathscr{A})$ is defined by $L_{n}=L \otimes I_{n}$ and obviously

$$
\Phi_{t n} \equiv \Phi_{t} \otimes I_{n}=\operatorname{expt} L_{n} \text {. }
$$

Differentiation of the inequality (2.6) for $\Phi_{t n}$ at $t=0$, noting that equality holds at $t=0$, gives

$$
L_{n}\left(X^{+} X\right)-L_{n}\left(X^{+*}\right) X-X^{+} L_{n}(X) \geqq 0
$$

for all $X \in M_{n}(\mathscr{A})$. From $\Phi_{t n}(I)=I$ and the fact that a positive map is a *-map follows $L_{n}(I)=0, L_{n}\left(X^{+}\right)=L_{n}(X)^{+}$. Introduce a dissipation function $D(L): \mathscr{A} \times$ $\mathscr{A} \rightarrow \mathscr{A}$ by

$$
D(L ; X, Y)=L\left(X^{+} Y\right)-L\left(X^{+}\right) Y-X^{+} L(Y) .
$$

Definition. If a bounded map $L: \mathscr{A} \rightarrow \mathscr{A}$ satisfies $L(I)=0, L\left(X^{+}\right)=L(X)^{+}$all $X \in \mathscr{A}$, and $D\left(L_{n} ; X, X\right) \geqq 0$ all $X \in M_{n}(\mathscr{A})$, all $n$, then $L$ is said to be completely dissipative. We write this $L \in \mathrm{CD}(\mathscr{A})$. Denote by $\mathrm{CD}(\mathscr{A})_{\sigma}$ the set of ultraweakly continuous elements in $\mathrm{CD}(\mathscr{A})$.

Remarks. 1. The conditions $L(I)=0$ and $L\left(X^{+}\right)=L(X)^{+}$extend to $L_{n}$.

2. $\mathrm{CD}(\mathscr{A})$ forms a convex cone.

3. There is the usual polarization identity such that $D(L)$ is determined by the diagonal values $D(L ; X, X)$.

Proposition 3. $D(L)$ determines $L$ up to a Hamiltonian.

Proof. If $D(L ; X, Y)=0$ for all $X, Y \in \mathscr{A}$ then $L$ is a derivation. We know that every derivation of a $W^{*}$-algebra is inner $[12,21]$. Hence there is a $Y \in \mathscr{A}$ such that $L(X)=[Y, X]$. But from $L\left(X^{+}\right)=L(X)^{+}$follows that $Y=i H$ where $H$ is s.a.

If $L(X)=i[H, X]$ then $\Phi_{t}$ can obviously be continued to a group of automorphisms $\Phi_{t}(X)=e^{i H t} X e^{-i H t}$. Conversely if $L$ generates a group of CP maps then both $L$ and $-L$ satisfy (3.1). Hence $D(L ; X, X)=0$ i.e. $D(L)=0$ and $L(X)=$ $i[H, X]$. Consequently $D \neq 0$ characterizes the lack of reversibility as well as the deviation of $\Phi_{t}$ from an automorphism.

In the following proposition we show the relation between (2.6) and (3.1) in a somewhat more general form than we need.

Proposition 4. Let $L: \mathscr{A} \rightarrow \mathscr{A}$ be a bounded *-map and put $\Phi_{t}=\operatorname{expt} L$. Then $(1) \Leftrightarrow(2)$ where

(1) $\Phi_{t}\left(X^{+}\right) \Phi_{t}(X) \leqq \Phi_{t}\left(X^{+} X\right) \quad$ all $\quad X \in \mathscr{A}, \quad \Phi_{t}(I)=I$

(2) $D(L ; X, X) \geqq 0 \quad$ all $X \in \mathscr{A}, \quad L(I)=0$. 
Proof. We have already noted that $(1) \Rightarrow(2)$. In order to show that $(2) \Rightarrow(1)$ we recall that $L$ generates a norm-contraction semigroup iff

$$
\Theta(L) \equiv \lim _{t \downarrow 0} t^{-1}(\|I+t L\|-1) \leqq 0
$$

(see [22] Theorem 2.1). From Corollary 1 of [20] we obtain

$$
\|I+t L\|=\sup _{U}\|U+t L(U)\|
$$

where the supremum is over all unitary elements in $\mathscr{A}$. But from (2) with $X=U$ follows that for $t \leqq \frac{1}{2}\|L\|^{-1}$

$$
\begin{aligned}
\|U+t L(U)\|^{2} & =\left\|I+t\left(L\left(U^{+}\right) U+U^{+} L(U)\right)+t^{2} L\left(U^{+}\right) L(U)\right\| \\
& \leqq\left\|I+t^{2} L\left(U^{+}\right) L(U)\right\| \leqq 1+t^{2}\|L\|^{2}
\end{aligned}
$$

i.e. $t^{-1}(\|I+t L\|-1) \leqq \frac{1}{2} t\|L\|^{2}$.

Hence $\Theta(L) \leqq 0$ and $L$ generates a contractive semigroup $\Phi_{t}$. Obviously $\Phi_{t}(I)=I$ and consequently $\left\|\Phi_{t}\right\|=1$. But from Corollary 1 of $[20]$ can then conclude that $\Phi_{t}$ is positive. [If we know that $L \in \mathrm{CD}(\mathscr{A})$ we can repeat the argument applied to $L_{n}$ and conclude that $\Phi_{t}$ is CP.] If $\Phi_{t}$ is positive, then so is

$$
S_{\lambda}=\lambda \int_{0}^{\infty} e^{-\lambda t} \Phi_{t} d t=\lambda(\lambda-L)^{-1}, \quad \lambda>0 .
$$

Note that $S_{\lambda}(I)=I$, and once more by Corollary 1 of [20] that $\left\|S_{\lambda}\right\|=1$. Now we can prove that

$$
S_{\lambda}\left(X^{+}\right) S_{\lambda}(X) \leqq S_{\lambda}\left(X^{+} X\right) \text {. }
$$

Let $Y=(\lambda-L)^{-1} X$. Then by (2)

$$
\begin{aligned}
S_{\lambda}\left(X^{+} X\right) & =S_{\lambda}\left[\lambda^{2} Y^{+} Y-\lambda\left(L\left(Y^{+}\right) Y+Y^{+} L(Y)\right)+L\left(Y^{+}\right) L(Y)\right] \\
& \geqq S_{\lambda}\left[\lambda(\lambda-L) Y^{+} Y\right]=\lambda^{2} Y^{+} Y=S_{\lambda}\left(X^{+}\right) S_{\lambda}(X) .
\end{aligned}
$$

We know that $([11]$ p. 341)

$$
\lim _{n \rightarrow \infty}\left\|\Phi_{t}-\Phi_{t}^{(n)}\right\|=0
$$

where $\Phi_{t}^{(n)} \equiv\left(S_{n / t}\right)^{n}$ satisfies (1) and so does $\Phi_{t}$ by continuity.

Theorem 1. Let $L$ be a bounded $*_{-m a p} \mathscr{A} \rightarrow \mathscr{A}$ and let $\Phi_{t}=\operatorname{expt} L$. Then $\Phi_{t} \in \mathrm{CP}(\mathscr{A})$, $\Phi_{t}(I)=I$ iff $L \in \mathrm{CD}(\mathscr{A})$.

Proof. Apply Proposition 4 to the extensions $L_{t n}$ and $\Phi_{t n}$ to $M_{n}(\mathscr{A})$ for every $n$.

Corollary 1. $\Phi_{t}$ is a norm continuous dynamical semigroup on $\mathscr{A}$ iff $\Phi_{t}=\operatorname{expt} L$ where $L \in \mathrm{CD}(\mathscr{A})_{\sigma}$.

It may be interesting to extend this theorem to semigroups of CP maps which do not satisfy the condition $\Phi_{t}(I)=I$.

Differentiation of the inequality (2.5) for $\Phi_{t n}$ at $t=0$ gives

$$
L_{n}\left(X^{+} X\right)+X^{+} L_{n}(I) X-L_{n}\left(X^{+}\right) X-X^{+} L_{n}(X) \geqq 0
$$

for all $X \in M_{n}(\mathscr{A})$. Define $L^{\prime}$ through

$$
L^{\prime}(X)=L(X)-\frac{1}{2}\{L(I), X\}
$$


where $\{X, Y\}=X Y+Y X$. If (3.2) holds for $L$ then (3.1) holds for $L^{\prime}$, consequently $L^{\prime} \in \mathrm{CD}(\mathscr{A})$. The map $L^{\prime \prime}(X)=\{K, X\}, K$ s.a. $\in \mathscr{A}$, generates the semigroup

$$
\exp t L^{\prime \prime}(X)=e^{t K} X e^{t K}
$$

and obviously $\exp t L^{\prime \prime} \in \mathrm{CP}(\mathscr{A})$. The semigroup generated by $L^{\prime}+L^{\prime \prime}, L^{\prime} \in \mathrm{CD}(\mathscr{A})$ is given by the Lie-Trotter formula

$$
\Phi_{t}=\exp \left[t\left(L^{\prime}+L^{\prime \prime}\right)\right]=\lim _{n \rightarrow \infty}\left[\exp \left(t L^{\prime} / n\right) \exp \left(t L^{\prime \prime} / n\right)\right]^{n}
$$

hence $\Phi_{t} \in \mathrm{CP}(\mathscr{A})$. We have arrived at

Corollary 2. Let $L$ be a bounded $*_{-m a p} \mathscr{A} \rightarrow \mathscr{A}$ and let $\Phi_{t}=\exp t L$. Then $\Phi_{t} \in \operatorname{CP}(\mathscr{A})$ iff (3.2) is satisfied for all $n$.

Remark. Proposition 4, Theorem 1, and Corollary 2 obviously hold when $\mathscr{A}$ is only assumed to be a $C^{*}$-algebra.

\section{General Form of Completely Dissipative Generators}

To begin with the assume that $\mathscr{A}$ is a hyperfinite factor in a separable Hilbert space $\mathscr{H}[23,24]$ which includes the case $\mathscr{A}=B(\mathscr{H})([25]$ p. 92). $\mathscr{A}$ is generated by a sequence of finite type $I_{n(p)}$ factors $\mathscr{A}_{p}$ such that $\mathscr{A}=\left(\bigcup \mathscr{A}_{p}\right)^{\prime \prime}, \mathscr{A}_{p} \subset \mathscr{A}_{p+1}$. The set of unitary elements in $\mathscr{A}_{p}$ is then a compact group with an invariant mean $\mathscr{M}_{p}$ defined by the Haar measure.

Proposition 5. If $L \in \mathrm{CD}(\mathscr{A})_{\sigma}$ then there is a $\Psi \in \mathrm{CP}(\mathscr{A})_{\sigma}$ and a s.a. $H \in \mathscr{A}$ such that for all $X \in \mathscr{A}$.

$$
L(X)=\Psi(X)-\frac{1}{2}\{\Psi(I), X\}+i[H, X] .
$$

Proof. Define $K_{p} \in \mathscr{A}$ through

$$
K_{p}=\mathscr{M}_{p}\left[L\left(U^{+}\right) U\right] .
$$
of $\mathscr{M}_{p}$

Obviously $\left\|K_{p}\right\| \leqq\|L\|$. For a unitary $V \in \mathscr{A}_{p}$ we have, due to the invariance

$$
\mathscr{M}_{p}\left[L\left(V U^{+}\right) U\right]=\mathscr{M}_{p}\left[L\left(U^{+}\right) U V\right]=K_{p} V .
$$
in $\mathscr{A}_{p}$

Hence, as every element in $\mathscr{A}_{p}$ is a finite linear combination of unitary elements

$$
\mathscr{M}_{p}\left[L\left(X U^{+}\right) U\right]=K_{p} X
$$

for all $X \in \mathscr{A}_{p}$. For $X, Y \in \mathscr{A}_{p}$ we find that

$$
\mathscr{M}_{p}[D(L ; U X, U Y)]=L\left(X^{+} Y\right)-K_{p} X^{+} Y-X^{+} Y K_{p}^{+} .
$$

Define the linear map $\Psi_{p}: \mathscr{A} \rightarrow \mathscr{A}$ by

$$
\Psi_{p}(X)=L(X)-K_{p} X-X K_{p}^{+} .
$$

Then as $L \in \mathrm{CD}(\mathscr{A})$, we have for $X \in \mathscr{A}_{p}$

$$
\Psi_{p}\left(X^{+} X\right)=\mathscr{M}_{p}[D(L ; U X, U X)] \geqq 0
$$


i.e. $\Psi_{p} \mid \mathscr{A}_{p}$ is positive. We can apply the same argument to $M_{n}\left(\mathscr{A}_{p}\right) \cong M_{n}(\mathbb{C}) \otimes \mathscr{A}_{p}$ and find $\mathscr{M}_{p n}\left[L_{n}\left(U^{+}\right) U\right]=K_{p} \otimes I_{n}$ where $\mathscr{M}_{p n}$ is the invariant mean on the group of unitary elements in $M_{n}\left(\mathscr{A}_{p}\right)$. From this it easily follows that

$$
\Psi_{p} \mid \mathscr{A}_{p} \in \mathrm{CP}\left(\mathscr{A}_{p}, \mathscr{A}\right) \text {. }
$$

Define

$$
\begin{aligned}
\Psi_{K}(X) & =L(X)-K X-X K^{+}, \quad K \in \mathscr{A} \\
\Gamma_{p} & =\left\{K \in \mathscr{A} ; \Psi_{K} \mid \mathscr{A}_{p} \in \operatorname{CP}\left(\mathscr{A}_{p}, \mathscr{A}\right),\|K\| \leqq\|L\|\right\} .
\end{aligned}
$$

As $K_{p} \in \Gamma_{p}$ we have that $\Gamma_{p} \neq \emptyset$, every $p$. Obviously $\Gamma_{p+1} \subset \Gamma_{p}$ and hence any finite intersection of $\Gamma_{p}$ 's is nonempty. Furthermore it is evident that $\Gamma_{p}$ is weakly closed.

As the unit ball in $\mathscr{A}$ is compact in the weak operator topology $\Gamma_{p}$ are weakly compact ([12] 1.3.4). Consequently $\Gamma=\bigcap \Gamma_{p} \neq \emptyset$.

Pick one $K \in \Gamma$. Then $\Psi=\Psi_{K}$ is CP on $\bigcup \mathscr{A}_{p}$. As $L$ is ultraweakly continuous, $\Psi$ is CP on $\left(\bigcup \mathscr{A}_{p}\right)^{\prime \prime}$. Obviously $\Psi(I)=-K-K^{+}$. Put $H=\frac{1}{2} i\left(K^{+}-K\right)$. The statement then follows.

Remark. The proof is easily modified to give the following statement. If $L: \mathscr{A} \rightarrow \mathscr{A}$ is a bounded ultraweakly continuous *-map such that $D(L, X, X) \geqq 0$ for all $X \in \mathscr{A}$ then there is a positive normal map $\Psi: \mathscr{A} \rightarrow \mathscr{A}$ and $K \in \mathscr{A}$ such that $L(X)=$ $\Psi(X)+K X+X K^{+}$. This result does not have any direct converse.

Now let $\mathscr{A}$ be any $C^{*}$-algebra.

Proposition 6. If $\Psi \in \mathrm{CP}(\mathscr{A}), H$ s.a. $\in \mathscr{A}$ then $L$ defined by (4.1) is in $\mathrm{CD}(\mathscr{A})$.

Proof. Obviously $L(I)=0$ and $L\left(X^{+}\right)=L(X)^{+}$. We know from Lemma 1 that $\Psi$ is of the form (2.3) where we can choose $\pi(I)=I$. Then

$$
\begin{aligned}
& \Psi\left(X^{+} X\right)+X^{+} \Psi(I) X-\Psi\left(X^{+}\right) X-X^{+} \Psi(X) \\
& =(\pi(X) V-V X)^{+}(\pi(X) V-V X) \geqq 0 .
\end{aligned}
$$

A simple rearrangement gives $D(L ; X, X) \geqq 0$.

The same argument applied to the extensions $\Psi_{n}$ and $L_{n}$ to $M_{n}(\mathscr{A})$ shows that $L \in \mathrm{CD}(\mathscr{A})$.

From Propositions 5, 6, and Lemma 2 follows

Theorem 2. $L \in \mathrm{CD}(\mathscr{H})_{\sigma}$ iff it is of the form

$$
L(X)=\sum\left(V_{j}^{+} X V_{j}-\frac{1}{2}\left\{V_{j}^{+} V_{j}, X\right\}\right)+i[H, X]
$$

where $V_{j}, \sum V_{j}^{+} V_{j} \in B(\mathscr{H}), H$ s.a. $\in B(\mathscr{H})$. The dual generator on the state space (Schrödinger picture) is of the form

$$
L(\varrho)=\frac{1}{2} \sum\left(\left[V_{j} \varrho, V_{j}^{+}\right]+\left[V_{j}, \varrho V_{j}^{+}\right]\right)-i[H, \varrho] .
$$

Corollary 1 of Theorem 1 and Theorem 2 give the explicit form of the generators of norm continuous quantum dynamical semigroups on $B(\mathscr{H})$.

Propositions 5, 6, and Corollary 2 of Theorem 1 give the following more general statement. 
Theorem 3. Let $\mathscr{A}$ be a $C^{*}$-algebra, $L: \mathscr{A} \rightarrow \mathscr{A}$ a bounded $*$-map and put $\Phi_{t}=$ exp tL. If $L(X)=\Psi(X)+K X+X K^{+}$where $K \in \mathscr{A}$ and $\Psi \in \mathrm{CP}(\mathscr{A})$, then $\Phi_{t} \in \mathrm{CP}(\mathscr{A})$.

Conversely if $\mathscr{A}$ is a hyperfine factor and if $\Phi_{t} \in \mathrm{CP}(\mathscr{A})_{\sigma}$, then $L$ is of this form with $\Psi \in \mathrm{CP}(\mathscr{A})_{\sigma}$.

\section{Convex Structure of the Completely Dissipative Generators}

It is tempting to interpret the form (4.1) of the CD generators as a decomposition into a Hamiltonian part and a dissipative part. Unfortunately this decomposition is not unique as can easily be seen from simple examples. It turns out to be convenient to study the equivalence classes of generators modulo Hamiltonians. In the case $\mathscr{A}=B(\mathscr{H})$ this gives a nice convex and partial order structure on the set of equivalence classes. We define an equivalence relation on $\operatorname{CD}(\mathscr{H})_{\sigma}$ by

$$
L \sim L^{\prime} \quad \text { iff } \quad D(L ; X, X)=D\left(L^{\prime} ; X, X\right) \text { for all } X \in B(\mathscr{H}) .
$$

Proposition 3 says that $L \sim L^{\prime}$ iff $L(X)=L^{\prime}(X)+i[H, X]$ for some s.a. $H \in B(\mathscr{H})$. In this case we also have $D\left(L_{n} ; X, X\right)=D\left(L_{n}^{\prime} ; X, X\right)$ all $X \in M_{n}(B(\mathscr{H}))$, all $n$.

We can now define the set of equivalence classes $\mathrm{CD}(\mathscr{H})_{\sigma}$ of $\mathrm{CD}$ generators modulo Hamiltonians. The equivalence class of $L$ is denoted $\tilde{L} \cdot \operatorname{CD}(\mathscr{H})_{\sigma}$ forms a convex cone. We introduce a partial order in $\operatorname{CD}(\mathscr{H})_{\sigma}$ by

$$
\tilde{L} \leqq \tilde{L}^{\prime} \quad \text { iff } \quad D\left(L_{n} ; X, X\right) \leqq D\left(L_{n}^{\prime} ; X, X\right) \text { for all } X \in M_{n}(B(\mathscr{H})) \text {, all } n \text {. }
$$

$D\left(L_{n}\right)$ is a function of $\tilde{L}$ alone, hence the definition is independent of the choice of representative element. Obviously $\tilde{L} \leqq \tilde{L}^{\prime}$ and $\tilde{L}^{\prime} \leqq \tilde{L}$ imply that $\tilde{L}=\tilde{L}^{\prime}$.

Definition. $\tilde{L}$ is pure (belongs to an extreme ray) in $\mathrm{CD}(\mathscr{H})_{\sigma}$ iff $\tilde{L}^{\prime} \leqq \tilde{L}$ implies that $\tilde{L}^{\prime}=\lambda \tilde{L}, \lambda \in[0,1]$.

Introduce the set of CP maps $\Psi_{X}$ defined by

$$
\Psi_{X}(Y)=D(L ; X, Y X)-D(L ; X, Y) X .
$$

Note that $\Psi_{X}$ determines $\tilde{L}$. The CP property follows from the CD property of $L$, but we can use (4.2) to obtain directly

$$
\Psi_{X}(Y)=\sum\left[V_{i}, X\right]^{+} Y\left[V_{i}, X\right] \equiv \sum \Psi_{i X}(Y) .
$$

If the sum in (5.1) contains terms not proportional to $\Psi_{X}$ then $\tilde{L}$ is obviously not pure. Conversely, if $\tilde{L}$ is not pure then $\Psi_{X}$ cannot be pure for all $X$. Hence $\tilde{L}$ is pure iff $\Psi_{X}$ are all pure. If $\Psi$ in (4.1) is pure then the $\Psi_{X}$ are all pure and so is $\tilde{L}$. Conversely, if $\tilde{L}$ is pure then in (5.1) we must have

$$
\Psi_{i X} \propto \Psi_{j X} \text { for all } i, j, X \text {. }
$$

Then we can choose $V$ such that (5.1) holds with a single $V_{i}=V$. Consequently $\Psi(X)=V^{+} X V$ satisfies (4.1). This proves

Theorem 4. The pure elements of $\mathrm{CD}(\mathscr{H})_{\sigma}$ ard precisely the equivalence classes of generators of the form (4.1) with pure $\Psi$.

Theorem 2 says that countable sums of such elements make up $\operatorname{CD}(\mathscr{H})_{\sigma}^{r}$. 
As special cases we can mention the generators of Gaussian semigroups.

$$
L(X)=V X V-\frac{1}{2}\left\{V^{2}, X\right\} \quad V \text { s.a., }
$$

and Poisson semigroups

$$
L(X)=V^{+} X V-X \quad V \text { unitary . }
$$

Kossakowski [8] treated these cases in detail.

Davies [26] gives a general theory of quantum semigroups subordinated to a classical convolution semigroup on $\mathbb{R}$ and derives the form of the generators from the classical Lévy-Khinchin formula. The Lévy-Khinchin formula says that the generator of a continuous convolution semigroup of measures on $\mathbb{R}$ is the sum of an integral over generators of Poisson semigroups, a Gaussian generator and a translation generator (which is the decomposition into pure elements in that case) [14]. We find that for quantum dynamical semigroups the pure elements in the set of generators are of a more general type than in the classical case, but contain the generators of Poisson and Gaussian semigroups as special cases. The most general form (4.2) of a generator as a sum over pure elements can then be considered to be a quantum analogue of the Lévy-Khinchin formula.

\section{Relations with Other Work}

The type of semigroup generators considered here is not new. Davies has treated generators of the form (4.1) (but in the Schrödinger picture) in his work on quantum stochastic processes $[26,27]$. There the generators were defined through a stochastic kernel which correspond to the map $\Psi$ in (4.1). As a special case Davies considered kernels which correspond to CP maps $\Psi$. The new point here is the claim that the restriction to semigroups of $\mathrm{CP}$ maps implies that the generators are of this simple form. Kossakowski has treated the general case of semigroups of positive maps. For a spin $\frac{1}{2}$ system the explicit form of the generators was given in [28]. In the case of an arbitrary Hilbert space necessary and sufficient conditions on the generators were derived in [29].

Equation (4.3) gives an explicit form for the most general time-homogenous quantum mechanical Markovian master equation with a bounded Liouville operator

$$
\varrho=L(\varrho) \text {. }
$$

It is an empirical fact that the Markovian master equations found in the literature turn out to be of this form after some rearrangement of the terms and allowing for unbounded operators. The conventional derivation of this type of master equation is either through a second order perturbation calculation with respect to the interaction of the considered system with the external reservoir [30] or by the projection technique introduced by Zwanzig [31-33]. In both cases the Markov approximation has to be postulated. The rigorous result by Davies (Eq. (4.9) and (4.19) of [5]) is once more a master equation with a Liouville operator of the form (4.3).

In [26] Theorem 5 it was shown that a semigroup generated by a master equation of this type can always be regarded as resulting from an interaction with 
a heat bath of a singular type. Adding the results of $\S 4$ we obtain the following statement which is formulated in the Schrödinger picture.

Proposition 9. Let $\Phi_{t}: S(\mathscr{H}) \rightarrow S(\mathscr{H})$ be a norm continuous quantum dynamical semigroup. Then there is a Hilbert space $\mathscr{H}^{\prime}$, a one-parameter isometric semigroup $V_{t}$ on $\mathscr{H}^{\prime}$ and trace-preserving linear maps $e: S(\mathscr{H}) \rightarrow S\left(\mathscr{H}^{\prime}\right)$ and $r: S\left(\mathscr{H}^{\prime}\right) \rightarrow S(\mathscr{H})$ such that

$$
\Phi_{t}(\varrho)=r\left(V_{t} e(\varrho) V_{t}^{+}\right) \quad \text { for all } \varrho \in S(\mathscr{H}) \text { and } t \geqq 0 \text {. }
$$

After the completion of this work we have recieved a preprint of a related work by V.Gorini et al. [34]. A result similar to Theorem 2 is derived for a system described by a finite-dimensional Hilbert space, using methods different from ours.

\section{References}

1. George, C., Prigogine,I., Rosenfeld,L.: Kgl. Danske Videnskab. Selskab Mat.-Fys. Medd 38, no 12 (1972)

2. Grecos, A. P., Prigogine, I.: Physica 59, 77-96 (1972)

3. Williams, D. N.: Commun. math. Phys. 21, $314-333$ (1971)

4. Davies, E. B.: Commun. math. Phys. 33, 171-186 (1973)

5. Davies, E. B.: Commun. math. Phys. 39, 81-110 (1974)

6. Pulè, J. V.: Commun. math. Phys. 38, 241-256 (1974)

7. Mehra, J., Sudarshan, E. C. G.: Nuovo Cimento 11 B, 215-256 (1972)

8. Kossakowski, A. : Rep. Math. Phys. 3, 247-274 (1972)

9. Ingarden, R.S., Kossakowski, A.: Ann. Phys. 89, 451-485 (1975)

10. Phillips, R.S.: Pacific J. Math. 5, 269-283 (1955)

11. Hille, E., Phillips, R. S. : Functional analysis and semigroups. Providence: Amer. Math. Soc. 1957

12. Dixmier, J.: Les algebres d'operateurs dans l'espace Hilbertien. Paris: Gauthier-Villars 1969

13. Stinespring, W. F.: Proc. Amer. Math. Soc. 6, 211-216 (1955)

14. Feller, W.: An introduction to probability theory and its applications, vol. 2. New York: Wiley 1971

15. Arveson, W.: Acta. Math. 123, 141-224 (1969)

16. Størmer, E.: Springer lecture notes in physics 29, 85-106 (1974)

17. Choi, M. D.: Canadian J. Math. 24, 520-529 (1972)

18. Kraus, K.: Ann. Phys. 64, 311-335 (1970)

19. Sz.-Nagy, B., Foias, C.: Harmonic analysis of operators on Hilbert space. Amsterdam: NorthHolland 1970

20. Russo, B., Dye, H. A.: Duke Math. J. 33, 413-416 (1966)

21. Kadison, R. V.: Bull. London Math. Soc. 7, $41-44$ (1975)

22. Lumer, G., Phillips, R. S. : Pacific J. Math. 11, 679-698 (1961)

23. Powers, R. T.: Ann. Math. 86, 138-171 (1967)

24. Sakai, S.: $C^{*}$-algebras and $W^{*}$-algebras. Berlin, Heidelberg; New York: Springer 1971

25. Topping, D. M.: Lectures on von Neumann algebras. London: Van Nostrand 1971

26. Davies, E. B.: Z. Wahrscheinlichkeitstheorie verw. Geb. 23, 261-273 (1972)

27. Davies, E. B.: Commun. math. Phys. 15, 277-304 (1969); 19, 83-105 (1970); 22, 51-70 (1971)

28. Kossakowski, A.: Bull. Acad. Polon. Sci. Ser. math. astr. et phys. 21, 649-653 (1973)

29. Kossakowski, A.: Bull. Acad. Polon. Sci. Ser. math. astr. et phys. 20, 1021-1025 (1972)

30. Haken, H.: Handb. Phys. 25, 2c. Berlin, Heidelberg, New York: Springer 1970

31. Zwanzig, R. : Physica 30, 1109-1123 (1964)

32. Emch, G. C., Sewell, G. L.: J. Math. Phys. 9, 846-958 (1968)

33. Haake, F... Springer Tracts Mod. Phys. 66, 98-168 (1973)

34. Gorini, V., Kossakowski, A., Sudarshan, E. C. G.: Preprint CPT 244, U. of Texas, Austin

Communicated by H. Araki

Received April 7, 1975; in revised form November 28, 1975 